\title{
EFFECT OF SPRAYING SOME NUTURAL COMPOUNDS AND AMINO ACIDA AS WELL AS PRUNING LATERAL BRANCHES ON GROWTH QUALITY, QUANTITY YIELD AND FRUIT QUALITY OF CANTALOUPE UNDER TUNNELS CONDITIONS.
}

Hasanein, N. M. ; S. M. Yuossef and Hala H. Abo El- Nour. Veg. Res. Dept. Hort. Res. Inst. Agric. Res. Center, Giza Egypt

\begin{abstract}
This research work was performed to study the interaction between some natural compounds, humic acids and pruning lateral branches of cantaloupe plants (Cucumus melo L.) cv Imperial were planting at Ali Moubarak Station,South Tahrir, El Behera Governorate during the two successive seasons of 2008\2009 and 2009\2010. The experimental design was split plot with four replicates having pruning lateral branches at reaction in the main plots and spraying some natural compounds, were randomly distributed in the sub plots. Each sub plot had one ridge with dimensions of $10 \mathrm{~m}$ long and $1 \mathrm{~m}$ width .Planting distance was $50 \mathrm{~cm}$ apart. Each sub plot was content on 20 plants.

Obtained results showed the superiority of without pruning plant treatments followed by the pruning lateral branches in terms of vegetative growth, fruit and yield characters. Using spraying garlic extract at a rate of $200 \mathrm{ppm} / \mathrm{feddan}$ or spraying amino acid at a rate of $200 \mathrm{ppm} /$ feddan increased plant content of $\mathrm{N}, \mathrm{P}, \mathrm{K}$ and some micro nutrients than the other treatments. In addition, the mentioned treatments improved the vegetative growth and fruit physical and chemical characters .Early and total yield were increased under spraying garlic or spraying sucrose at a rate of 500 ppmlfeddan. Lowest vegetative growth, fruit and yield characters were obtained from that without spraying treatments (control).
\end{abstract}

Kaywords: Pruning lateral branches, spraying garlic extract, spraying amino acid, spraying sucrose.

\section{INTRODUCTION}

Melon (Cucumis melo L.) is an important horticultural crop, often cultivated in arid and semi-arid regions of the world; Melon fruit enjoys widespread popularity among consumers owing to its taste and high nutritional value. (Joanna, 2009). Amino acids which account for the majority of organic nitrogen in soils, impact plant growth and serve to explain how organic matter promotes soil productivity (Schnitzer, 2001). However, foliar nutrition in the form of amino acids provides readymade building blocks for protein synthesis. The amino acid is absorbed by the cells as such, and is simply fed into the metabolic machinery of the cell .Amino acids can directly or indirectly influence the physiological activates of the plant .Because of the amino acid pool is only a small portion of the total dissolved organic nitrogen pool, which generally contains less than $10 \%$ free amino acids in temperate ecosystems (Qualls and Haines 1991 and Yu et al., 2002). Plants can up take $\mathrm{N}$ in forms of amino acids without relying on microbial mineralization (Lipson and Nasholm, 2001). Amino acids help to increase chlorophyll concentration in plant leading to higher degree of photosynthesis.which lead to increase carbohydrates content they comprise structurally polysaccharides of plant, 
principally cellulose, hemicelluloses and pectin and lignin which consider an important structural compound of plant such improvement in growth parameters and yield (Hahlbrock and Scheel, 1989). Foliar application with proline and glycine at a rate of $100 \mathrm{ppm}$ has simulative effect on the number of bean pods per plant and total yield under low tunnels. (Ahmad et al., 2006). Spraying amino acid (AG) at a rate of $200 \mathrm{ppm}$ had a significant earlier and higher watermelon total yield than the control tap water (Hasanin and Gaafer, 2006). The highest N, P and K content in seed and straw of faba bean were observed as response to amino and humic acids application. (Ayman et al., 2009). Nevertheless, the majority of all amino and humic acids treatments significantly increased growth parameters measuring height, branches and leaves number per plant. The greatest potato stem height, number of leaves per plant and tuber yield was achieved with the extract of garlic bulbs comparing with the control in winter and summer growing seasons (Abd El-Khair and Haggag, 2007). Spraying garlic or onion extracts at concentration of $9 \%$ increased length, fresh and dry weight of shoots and roots as well as number of flowers/plant compared with control. (Morsy et al., 2009).

Concerning the amino acid, the role of amino acid in stimulating the growth and activating plants were studied by Phillips (1971). Who stated that available evidence suggested that several alternative routes of IAA synthesis exist in plants all starting from amino acids. Koriesh (1984) and Harridy (1986) noticed that the highest values of fresh and dry weights, as well as alkaloids content of Catharantins plants were observed when sprayed by tryptophan at $100 \mathrm{ppm}$. The same results were reported by Mohamed et al. (1992) on Alpinia and Osman (1997) on narcissus plants. In addition, Sutha et al. (1998) reported that an increase in protein content with significant increase in amino acid concentrations was noticed in tomato plants. However, Eppendorfer and Bille (1996) and El-Shabasi et al. (2005) indicated that the concentration of total amino acids in dry matter (DM) increased linearly with increasing total $\mathrm{N}$ content in potato tuber. Moreover, Hasanein and Gaafer (2006) indicated that application of amino acids (AG) spraying at a rate of $200 \mathrm{ppm}$ alone was increased vegetative growth and higher early and total yield on watermelon plants. In addition, Lansbergen et al. (2006) on cucumber plants reported that thinning one quarter of fruits before they each the wires was the most effective resulting in adequate regulation of the plants and the greatest increase in yield compared with the control .It is concluded that thinning of cucumber fruits is a viable alternative for increasing productivity and profitability.

Concerning garlic extract application to the culture it gave high reduction or inhibition of enzymatic activities. The growth of the fungi species was remarkably reduced by the garlic extract. (Tawfik et al., 2005).

The aim of the present study was to investigate the effect of some natural compounds on some physical properties of sandy soil and yield of cantaloupe plants.

\section{MATERIALS AND MEHTODS}


This study was performed to study the effect of spraying with some natural compounds and pruning lateral branches of cantaloupe plants at Ali Moubarak station, South Tahrir, El Behera governorate during two successive seasons, i.e., 2008/2009 and 200912010. The experiment design was split plot with four replicates, Having pruning lateral branches in the main plot and spraying some natural compounds which were randomly distributed in the sub plots. Each experimental plot consisted of one bad 10 meters long and one meter width and it contained 20 plants of Cantaloupe (Cucumis Melo L.) cv. Imperial hybrid. Seeds were sown on October $15^{\text {th }} 2008$ and on October $19^{\text {th }} 2009$ in a nursery. Seedling transplanting took place on November $6^{\text {th }}$ 2008 and on November $11^{\text {th }} 2009$ with using polyethylene low tunnels.

\section{Treatments:-}

A- Main plots.

1- Using pruning lateral branches (Three lateral branches).

2- Without pruning lateral branches.

\section{B- Sub main plots.}

Spraying Natural compound treatments as follows:-.

1 - Spraying garlic extract at a rate of $200 \mathrm{ppm} / \mathrm{feddan}$.

2- Spraying amino acids contents of free amino acids i.e., Glutamic, Aspartic and Lysine at a rate of $200 \mathrm{ppm} / \mathrm{feddan}$.

3- Spraying sucrose at a rate of $200 \mathrm{~g} / 1000 \mathrm{ml}$.

4- without spraying (Control).

Chicken manure was applied 20 days before planting at $20 \mathrm{~cm}$ depth from the soil surface. All the experimental plots received the same amount of fertigate water from planting till harvest using drip irrigation system. The distance between plants was $50 \mathrm{~cm}$. All plants received the spraying treatments three times.The first one was 30 days after planting, the second and third were performed after 21 days intervals with equal amount for each application. The spraying was carried out at the early morning. Physical and chemical analyses of the soil are presented in Table 1. 200 grams garlic cloves were washed under sterile distilled water and blotted with paper towels .Samples were cut into small pieces and added to $1000 \mathrm{ml}$ distilled water and then macerated by blender (for 10 minuet at room temperature . The extracts were obtained by centrifuging samples). Sorvall RC5B centrifuge, Newton, CT for 45 minuets to remove bigger particles. The supernatants were collected and sterilized using a Nalgene filter diameter $0.42 \mathrm{~mm}$, Fisher Scientific.

Table 1: The Physical and chemical properties of the experimental soil.

\begin{tabular}{|c|c|c|c|c|c|c|c|c|c|c|c|c|c|c|}
\hline \multicolumn{15}{|c|}{$2008 / 2009$} \\
\hline \multicolumn{3}{|c|}{$\begin{array}{l}\text { Particle size } \\
\text { distribution }\end{array}$} & \multirow[t]{2}{*}{ Texture } & \multirow[t]{2}{*}{$\mathrm{P}^{\mathrm{H}}$} & \multirow{2}{*}{$\begin{array}{l}\mathrm{EC} \\
\mathrm{dS} / \mathrm{m}\end{array}$} & \multirow{2}{*}{$\begin{array}{l}\mathrm{C} / \mathrm{N} \\
\text { ratio }\end{array}$} & \multirow{2}{*}{$\begin{array}{c}\mathrm{CaCo}_{3} \\
\%\end{array}$} & \multirow{2}{*}{$\begin{array}{l}\text { OM } \\
\%\end{array}$} & \multicolumn{6}{|c|}{$\begin{array}{c}\mathrm{mg} / \mathrm{kg} \\
\text { Soil }\end{array}$} \\
\hline Sand & Silt & Clay & & & & & & & $\mathbf{N}$ & $\mathbf{P}$ & $\mathbf{K}$ & $\mathrm{Fe}$ & Zn & Mn \\
\hline 90.37 & 2.13 & 7.50 & $\begin{array}{l}\text { Loamy } \\
\text { sand }\end{array}$ & 7.4 & 1.25 & $1: 19$ & 2.74 & 0.57 & 9.7 & 13.6 & 92.1 & 2.19 & 1.28 & 1.27 \\
\hline \multicolumn{15}{|c|}{010} \\
\hline 91.65 & 1.95 & 6.40 & L. Sand & 7.2 & 1.18 & $1: 21$ & 267 & 5 & 9.3 & 12.8 & 95.1 & 2.27 & 1.32 & 1.24 \\
\hline
\end{tabular}

The extracts were then kept at $4 \mathrm{C}^{0}$ until use within $24 \mathrm{~h}$. Then, This concentration (200 ppm) as a crude aqueous extract, Balestra et al. (2009) . 
A random sample of three plants was taken from each plot at 120 days after planting for the determinations of the following measurements:

A- Physical characteristics.

1- Plant length $(\mathrm{cm})$.

2- Plant fresh weight: the average fresh weights of plants and fruits were determined in $(\mathrm{g})$ using digital electrical balance.

3- Plant dry weight: the average dry weight $(\mathrm{g})$ of plants sample was dried at $70 \mathrm{C}^{0}$.

4- Number of leaves per plant.

5 - Leaf area the average leaf area $\left(\mathrm{cm}^{2}\right)$ was measured of the $5^{\text {th }}$ true leaf by using laser leaf area meter.

6- Fruit length and diameter $(\mathrm{cm})$ : the average length (from the blossom and to the leaf peduncle) and diameter of fruits were determined.

7- Shape index fruit length/diameter (L/D).

8- Flesh thickness (cm).

B- Chemical characteristics.

1- Total soluble solid (TSS) was determined by using referactometer AOAC (1991)

2- Mineral content in vegetative growth were determined as follow:-

a- Nitrogen content as $\mathrm{g} / \mathrm{l} 00 \mathrm{~g}$ dry weight was assayed according to Jackson (1973) using the microkjeldahl apparatus.

b- Phosphorus content was determined calorimetrically as $\mathrm{g} / 100 \mathrm{~g}$ dry weight according to the method described by Jackson (1973).

$\mathrm{c}-\mathrm{K}, \mathrm{Fe}$ and $\mathrm{Mn}$ contents were determined by using atomic absorption spectrophotometer (Barkin Elmer 3300) according to Chiappmann and Pratt (1973), the results calculated as gl100g dry weight for $\mathrm{K}$ as ppm for $\mathrm{Fe}$ and $\mathrm{Mn}$.

C- Yield and its components:

1- Early fruit yield $\mathrm{kg}$ per plant.

2- Total fruit yield kg per plant.

3- Number of fruit per plant.

4- Average fruit weight (g).

5- Total fruit yield ton per feddan.

Statistical analysis:-

Data were subjected to statistical analysis using the analysis of variance method the means of treatments were compared by using the least significant difference (LSD) at 0.05 level of probability according to Snedecor and Cochran (1967).

\section{RESULTS AND DISCUSSION}


Vegetative growth of cantaloupe plant leaves grown under low tunnels condition.

Without punning lateral branches gave significantly higher plant height, leaf area, number of leaves per plant, fresh and dry weight than pruning lateral branches with significant effect of some natural compound and amino acids. Results in Table 2 showed that spraying garlic at a rate of 200 $\mathrm{ppm} / \mathrm{feddan}$ increased higher plant, leaf area, and number of leaves per plant, fresh and dry weight than the other treatments with significant differences.

The second treatments that increased the vegetative growth parameters were spraying amino acids at a rate of $200 \mathrm{ppm} / \mathrm{feddan}$. Similar results were obtained by Phillips (1971), Schnitzer (2001) and Joanna (2009).

Table 2: Effect of spraying for some natural compound, amino acids and pruning lateral branches on vegetative growth of cantaloupe plants grown under low tunnels conditions during $2008 / 2009$ and $2009 / 2010$ seasons.

\begin{tabular}{|c|c|c|c|c|c|c|c|c|c|c|}
\hline \multirow{3}{*}{ Characters } & \multicolumn{5}{|c|}{$2008 / 2009$} & \multicolumn{5}{|c|}{$2009 / 2010$} \\
\hline & \multirow{2}{*}{\begin{tabular}{|c} 
plant \\
height \\
cm
\end{tabular}} & \multirow{2}{*}{$\begin{array}{l}\text { Leaf } \\
\text { area } \\
\mathrm{cm}^{2} \\
\end{array}$} & \multirow{2}{*}{$\begin{array}{l}\text { No. of } \\
\text { leaves } \\
\text { /plant }\end{array}$} & \multirow{2}{*}{$\begin{array}{c}\text { Fresh } \\
\text { weight } \\
\text { g }\end{array}$} & \multirow{2}{*}{$\begin{array}{c}\text { Dry } \\
\text { weigh } \\
\text { g }\end{array}$} & \multirow{2}{*}{\begin{tabular}{|c|}
$\begin{array}{c}\text { Plant } \\
\text { height } \\
\text { cm }\end{array}$ \\
\end{tabular}} & \multirow{2}{*}{$\begin{array}{l}\text { Leaf } \\
\text { area } \\
\text { cm }^{2}\end{array}$} & \multirow{2}{*}{$\begin{array}{l}\text { No. of } \\
\text { leaves } \\
\text { /plant }\end{array}$} & \multirow{2}{*}{$\begin{array}{c}\text { Fresh } \\
\text { weight } \\
\text { g }\end{array}$} & \multirow{2}{*}{$\begin{array}{c}\text { dry } \\
\text { weight } \\
\text { g }\end{array}$} \\
\hline & & & & & & & & & & \\
\hline \begin{tabular}{|l|l|} 
A & PLB \\
\end{tabular} & 162.6 & 151.0 & 68.4 & 688.8 & 157.0 & 156.3 & 147.8 & & & 150.6 \\
\hline & & & & & & & 152.9 & & & \\
\hline F. Test & * & * & * & & & & & & & \\
\hline \begin{tabular}{l|l} 
B & SP
\end{tabular} & 36.0 & 169.2 & 82.9 & & 74.9 & & 165.9 & 78 & & 168.5 \\
\hline SAA & & $15 \varsigma$ & 75.3 & & & & 156.6 & & & \\
\hline SS & 8 & 139 & 58.3 & & 144.5 & 144 & 133.6 & 55.6 & & 141.0 \\
\hline C & 158.4 & 147.2 & 65.2 & 658 & 154.8 & 153.4 & 145.4 & 64.1 & 661.5 & 147.3 \\
\hline SD at $5 \%$ & 6.4 & 3.2 & 4.6 & 17.1 & 8.6 & & & 2.7 & 21.7 & 0.9 \\
\hline
\end{tabular}

PLB = pruning lateral branches W. PLB = without pruning lateral branches

$\mathrm{SP}=$ Spraying garlic extract at a rate of $200 \mathrm{ppm} / \mathrm{fed}$.

SAA = Spraying amino acid at a rate of $200 \mathrm{ppm} / \mathrm{fed}$.

SS = Spraying sucrose at a rate of $500 \mathrm{ppm} / \mathrm{fed}$.

$\mathrm{C}=$ without spraying (Control).

The lowest vegetative growth was obtained from spraying sucrose at a rate of $500 \mathrm{ppm} / \mathrm{feddan}$. It can be concluded from the results that spraying garlic increased the vegetative growth.

The interaction of some natural compound, amino acids and pruning lateral branches on vegetative growth are presented in Table 3 . Results showed that without pruning lateral branches and spraying garlic increased the vegetative growth i.e., the values higher plant, leaf area, number of leaves per plant, fresh and dry weight was significantly greater than the other treatments during the two studied seasons.

Table 3: Effect of spraying for some natural compound, amino acids and pruning lateral branches interaction on vegetative growth of cantaloupe plants grown under low tunnels conditions during 2008/2009 and 2009/2010 seasons. 
Hasanein, N. M. et al.

\begin{tabular}{|c|c|c|c|c|c|c|c|c|c|c|c|}
\hline \multicolumn{2}{|c|}{ Treatments } & \multicolumn{5}{|c|}{$2008 / 2009$} & \multicolumn{5}{|c|}{$2009 / 2010$} \\
\hline Main & $\begin{array}{l}\text { sub } \\
\text { main }\end{array}$ & $\begin{array}{c}\text { Plant } \\
\text { height } \\
\text { cm }\end{array}$ & $\begin{array}{l}\text { Leaf } \\
\text { area } \\
\mathrm{cm}^{2}\end{array}$ & $\begin{array}{c}\text { No. of } \\
\text { leaves } \\
\text { /plant }\end{array}$ & $\begin{array}{c}\text { Fresh } \\
\text { weight } \\
\text { g }\end{array}$ & $\begin{array}{c}\text { Dry } \\
\text { weight } \\
\text { g }\end{array}$ & $\begin{array}{c}\text { Plant } \\
\text { height } \\
\text { cm }\end{array}$ & $\begin{array}{l}\text { Leaf } \\
\text { area } \\
\mathrm{cm}^{2}\end{array}$ & $\begin{array}{c}\text { No. of } \\
\text { leaves } \\
\text { /lant }\end{array}$ & $\begin{array}{c}\text { fresh } \\
\text { weight } \\
\text { g }\end{array}$ & $\begin{array}{c}\text { dry } \\
\text { weight } \\
\text { g }\end{array}$ \\
\hline \multirow[t]{4}{*}{ PLB } & SP & 182.6 & 166.6 & 81.1 & 745 & 172.6 & 175.1 & 164.5 & 76.6 & 727 & 166.6 \\
\hline & SAA & 165.7 & 155.7 & 72.8 & 719 & 160.4 & 158.9 & 153.9 & 69.2 & 697 & 158.7 \\
\hline & SS & 145.9 & 137.4 & 56.2 & 666 & 141.2 & 140.7 & 130.8 & 53.8 & 609 & 137.8 \\
\hline & C & 156.3 & 144.3 & 63.4 & 625 & 153.8 & 150.4 & 142.1 & 61.7 & 653 & 139.1 \\
\hline W. & SP & 189.3 & 171.8 & 84.6 & 763 & 177.1 & 182.6 & 167.2 & 80.1 & 741 & 170.3 \\
\hline \multirow[t]{3}{*}{ PLB } & SAA & 173.1 & 162.9 & 77.7 & 732 & 167.3 & 170.3 & 159.3 & 73.9 & 708 & 163.9 \\
\hline & SS & 151.6 & 141.2 & 60.3 & 641 & 147.9 & 147.2 & 136.4 & 57.3 & 627 & 144.2 \\
\hline & C & 160.4 & 150.1 & 66.9 & 696 & 155.7 & 156.3 & 148.6 & 66.4 & 670 & 155.5 \\
\hline \multicolumn{2}{|c|}{ LSD at $5 \%$} & 4. 4 & 2.2 & 1.4 & 12.7 & 7.8 & 3.0 & 1.5 & 0.7 & 9.1 & 6.4 \\
\hline
\end{tabular}

PLB = pruning lateral branches W. PLB = without pruning lateral branches

$\mathrm{SP}=$ Spraying garlic extract at a rate of $200 \mathrm{ppm} / \mathrm{fed}$.

SAA = Spraying amino acid at a rate of $200 \mathrm{ppm} / \mathrm{fed}$.

$\mathrm{SS}=$ Spraying sucrose at a rate of $500 \mathrm{ppm} / \mathrm{fed}$.

$\mathrm{C}=$ without spraying (Control).

The lowest vegetative growth was obtained from pruning lateral branches during the two seasons. From the mentioned results it can be concluded that spraying garlic at a rate of $200 \mathrm{ppm} / \mathrm{fed}$ and without pruning lateral branches increased the vegetative growth compared with the other treatments and control .Similar results were obtained by Koriesh (1984), Harridy (1986), Hasanein and Gaafer (2006), Abd El-Khair and Haggag (2007) and Ayman et al. (2009).

1- Physical and chemical characteristics of cantaloupe fruits grown under low tunnels conditions.

Table (4) show the effect of some natural compound, amino acids and pruning lateral branches on fruit physical characteristics of cantaloupe grown under low tunnels. It is clear that pruning lateral branches showed higher values of fruit length, diameter, flesh thickness and TSS\% than without pruning lateral branches with significant differences during the two studied seasons.

Concerning, some natural compound and amino acids the results indicated that spraying sucrose at a rate of $500 \mathrm{ppm} / \mathrm{fed}$. increased fruit physical characteristics compared with the other treatments and control .The results were conformed in two seasons and agreed with those obtained by Mohamed et al. (1992), Osman (1997), Sutha et al. (1998)and Lipson and Nasholm ( 2001).

Table 4: Effect of spraying for some natural compound, amino acids and pruning lateral branches on fruit characteristics of cantaloupe plants grown under low tunnels conditions during $2008 / 2009$ and $2009 / 2010$ seasons.

\begin{tabular}{|l|l|l|}
\hline Charaeters & $2008 / 2009$ & $2009 / 2010$ \\
\hline
\end{tabular}




\begin{tabular}{|c|c|c|c|c|c|c|c|c|c|c|c|}
\hline & & \begin{tabular}{|c|} 
fruit \\
length \\
$\mathrm{cm}$
\end{tabular} & \begin{tabular}{|c|} 
Fruit \\
diameter \\
cm
\end{tabular} & $\begin{array}{l}\text { Shape } \\
\text { index }\end{array}$ & \begin{tabular}{|c|} 
Fresh \\
thickness \\
cm
\end{tabular} & $\begin{array}{c}\text { TSS } \\
\%\end{array}$ & $\begin{array}{c}\text { Fruit } \\
\text { length } \\
\mathrm{cm}\end{array}$ & \begin{tabular}{|c|} 
Fruit \\
diameter \\
cm
\end{tabular} & $\begin{array}{l}\text { shape } \\
\text { index }\end{array}$ & \begin{tabular}{|c|} 
Fresh \\
thickness \\
cm
\end{tabular} & TSS \\
\hline \multirow[t]{2}{*}{ A } & PLB & 9.6 & 12.9 & 0.737 & 3.7 & 14.0 & 9.3 & 13.3 & 0.693 & 3.4 & 13.6 \\
\hline & W. IB & 9.1 & 12.5 & & 3.4 & 13.7 & 8.9 & 13.0 & & 3.1 & 13.3 \\
\hline \multicolumn{2}{|c|}{ F. Test } & * & * & * & * & * & * & * & * & * & * \\
\hline \multirow[t]{4}{*}{$\mathbf{B}$} & SP & 8.0 & 11.3 & 0.706 & 2.7 & 13.0 & 7.8 & 11.9 & 0.658 & 2.4 & 12.6 \\
\hline & SAA & 9.9 & 13.1 & 0.748 & 3.9 & 14.2 & 9.7 & 13.6 & & 3.5 & 13.7 \\
\hline & sS & 10.5 & 13.9 & 0.761 & 4.4 & 14.7 & 10.2 & 14.3 & & 4.1 & 14.4 \\
\hline & C & 9.0 & 12.5 & 0.720 & 3.3 & 13.6 & 8.8 & 13.0 & 0.675 & 3.0 & 13.3 \\
\hline \multicolumn{2}{|c|}{ LSD at $5 \%$} & 0.4 & 0.5 & 0.01 & 0.1 & 0.3 & 0.3 & 0.6 & 0.02 & 0.2 & 0.4 \\
\hline
\end{tabular}

PLB = pruning lateral branches $\quad$ W. PLB = without pruning lateral branches

$\mathrm{SP}=$ Spraying garlic extract at a rate of $200 \mathrm{ppm} / \mathrm{fed}$.

$\mathrm{SAA}=$ Spraying amino acid at a rate of $200 \mathrm{ppm} / \mathrm{fed}$.

$\mathrm{SS}=$ Spraying sucrose at a rate of $500 \mathrm{ppm} / \mathrm{fed}$.

$\mathrm{C}=$ without spraying (Control).

The lowest fruit physical (Length, diameter, shape index and fresh thickness) value were obtained from spraying garlic during the two seasons. In addition, spraying amino acids improved the fruit physical characteristics i.e., Fruit length, diameter, flesh thickness and TSS\% than the other treatments and control during the two seasons 2008/2009 and 2009/2010.

The results of the interaction between the effect of some natural compound, amino acids and pruning lateral branches on the fruit physical characteristics are presented in Table 5.

Table 5: Effect of spraying for some natural compound, amino acids and pruning lateral branches interaction on fruit characteristics of cantaloupe plants grown under low tunnels conditions during 2008/2009 and 2009/2010 seasons.

\begin{tabular}{|c|c|c|c|c|c|c|c|c|c|c|c|}
\hline \multicolumn{2}{|c|}{ Treatments } & \multicolumn{5}{|c|}{$2008 / 2009$} & \multicolumn{5}{|c|}{$2009 / 2010$} \\
\hline Main & $\begin{array}{l}\text { sub } \\
\text { main }\end{array}$ & $\begin{array}{c}\text { Fruit } \\
\text { length } \\
\text { cm }\end{array}$ & \begin{tabular}{|c|} 
Fruit \\
diameter \\
$\mathbf{c m}$
\end{tabular} & $\begin{array}{l}\text { Shape } \\
\text { index }\end{array}$ & $\begin{array}{c}\text { Fresh } \\
\text { thickness } \\
\mathrm{cm}\end{array}$ & $\begin{array}{c}\text { TSS } \\
\%\end{array}$ & $\begin{array}{c}\text { Fruit } \\
\text { length } \\
\text { cm }\end{array}$ & \begin{tabular}{|c} 
Fruit \\
diameter \\
$\mathbf{c m}$
\end{tabular} & $\begin{array}{l}\text { shape } \\
\text { index }\end{array}$ & $\begin{array}{c}\text { Fresh } \\
\text { thickness } \\
\text { cm }\end{array}$ & $\begin{array}{r}\text { TSS } \\
\%\end{array}$ \\
\hline PLB & SP & 8.2 & 11.6 & 0.706 & 2.9 & 13.2 & 8.0 & 12.1 & 0.661 & 2.6 & 12.8 \\
\hline & SAA & 10.1 & 13.1 & 0.759 & 4.0 & 14.3 & 9.8 & 13.8 & 0.710 & 3.6 & 13.8 \\
\hline & SS & 10.7 & 14.1 & 0.756 & 4.5 & 14.8 & 10.3 & 14.4 & 0.715 & 4.2 & 14.5 \\
\hline & C & 9.2 & 12.7 & 0.726 & 3.5 & 13.7 & 9.1 & 13.2 & 0.685 & 3.1 & 13.4 \\
\hline W. PLE & SP & 7.8 & 11.0 & 0.705 & 2.5 & 12.8 & 7.6 & 11.6 & 0.655 & 2.1 & 12.4 \\
\hline & SAA & 9.6 & 13.0 & 0.736 & 3.8 & 14.0 & 9.5 & 13.4 & 0.706 & 3.4 & 13.6 \\
\hline & SS & 10.4 & 13.6 & 0.785 & 4.3 & 14.5 & 10.0 & 14.1 & 0.705 & 4.0 & 14.2 \\
\hline & C & 8.7 & 12.2 & 0.713 & 3.1 & 13.5 & 8.5 & 12.7 & 0.665 & 2.9 & 13.1 \\
\hline LSD at & $5 \%$ & 0.2 & 0.3 & N.S & 0.2 & 0.1 & 0.3 & 0.2 & N.S & 0.2 & 0.3 \\
\hline
\end{tabular}

PLB = pruning lateral branches $\quad$ W. PLB = without pruning lateral branches

$\mathrm{SP}=$ Spraying garlic extract at a rate of $200 \mathrm{ppm} / \mathrm{fed}$.

SAA $=$ Spraying amino acid at a rate of $200 \mathrm{ppm} / \mathrm{fed}$.

$\mathrm{SS}=$ Spraying sucrose at a rate of $500 \mathrm{ppm} / \mathrm{fed}$

$\mathbf{C}=$ without spraying (Control).

Data in Table 5 show that physical fruit values were significantly increased under spraying sucrose and amino acids treatments compared with the other treatments during the two studied seasons. The results were conformed in two seasons. 
1- Effect of some natural compound, amino acids and pruning lateral branches interaction on yield productivity of cantaloupe fruits grown under low tunnels condition.

Early yield, total yield, average fruit weight and total yield (ton/fed.) of cantaloupe fruits influenced by different natural compound and pruning lateral branches are presented in Table 6.

Table 6: Effect of spraying for some natural compound, amino acids and pruning lateral branches on yield and its components of cantaloupe plants grown under low tunnels conditions during $2008 / 2009$ and $2009 / 2010$ seasons.

\begin{tabular}{|c|c|c|c|c|c|c|c|c|c|c|c|}
\hline \multirow{2}{*}{\multicolumn{2}{|c|}{ h }} & \multicolumn{5}{|c|}{$2008 / 2009$} & \multicolumn{5}{|c|}{$2009 / 2010$} \\
\hline & & $\begin{array}{c}\text { early } \\
\text { yield } \\
\text { kg/ } \\
\text { plant }\end{array}$ & $\begin{array}{c}\text { No. of } \\
\text { Fruit/P } \\
\text { lant }\end{array}$ & $\begin{array}{c}\text { total } \\
\text { yield } \\
\text { kg/ } \\
\text { plant }\end{array}$ & $\begin{array}{c}\text { Average } \\
\text { fruit } \\
\text { weight } \\
\text { g }\end{array}$ & $\begin{array}{l}\text { Total } \\
\text { yield } \\
\text { ton/ } \\
\text { fed. }\end{array}$ & $\begin{array}{c}\text { Early } \\
\text { yield } \\
\text { kg/ } \\
\text { plant }\end{array}$ & $\begin{array}{l}\text { No. of } \\
\text { Fruit/ } \\
\text { plant }\end{array}$ & \begin{tabular}{|l} 
total \\
yield \\
kg/ \\
plant
\end{tabular} & $\begin{array}{c}\text { average } \\
\text { fruit } \\
\text { weight } \\
\text { g }\end{array}$ & $\begin{array}{l}\text { total } \\
\text { yield } \\
\text { ton/ } \\
\text { fed. }\end{array}$ \\
\hline \multirow[t]{2}{*}{$\mathbf{A}$} & PLB & 3.9 & 9.8 & 8.0 & 823.8 & 20.1 & 4.0 & 9.7 & 8.2 & 846.0 & 20.4 \\
\hline & W. PLB & 3.8 & 9.9 & 7.9 & 798.5 & 19.7 & 3.9 & 9.8 & 8.0 & 821.3 & 19.9 \\
\hline \multicolumn{2}{|c|}{ F. Test } & NS & NS & NS & ${ }^{*}$ & ${ }^{*}$ & NS & NS & NS & ${ }^{*}$ & ${ }^{*}$ \\
\hline \multirow[t]{4}{*}{$\mathbf{B}$} & SP & 3.4 & 10.1 & 7.5 & 741.5 & 18.7 & 3.5 & 10.0 & 7.6 & 759.5 & 18.9 \\
\hline & SAA & 4.0 & 9.7 & 8.2 & 839.5 & 20.4 & 4.1 & 9.5 & 8.2 & 865.0 & 20.6 \\
\hline & SS & 4.2 & 9.6 & 8.4 & 882.5 & 21.0 & 4.4 & 9.5 & 8.6 & 900.5 & 21.4 \\
\hline & C & 3.7 & 10.1 & 7.8 & 781.5 & 19.6 & 3.9 & 9.8 & 7.9 & 809.5 & 19.8 \\
\hline \multicolumn{2}{|c|}{ LSD at $5 \%$} & 0.1 & NS & 0.1 & 4.4 & 0.4 & 0.2 & NS & 0.2 & 26.3 & 0.5 \\
\hline
\end{tabular}

PLB = pruning lateral branches $\quad$ W. PLB = without pruning lateral branches

$\mathrm{SP}=$ Spraying garlic extract at a rate of $200 \mathrm{ppm} / \mathrm{fed}$.

SAA $=$ Spraying amino acid at a rate of $200 \mathrm{ppm} / \mathrm{fed}$.

$\mathrm{SS}=$ Spraying sucrose at a rate of $500 \mathrm{ppm} / \mathrm{fed}$

C= without spraying (Control).

Data reveal that pruning lateral branches gave higher early yield, total yield, average fruit weight and total yield (ton/fed.) under spraying sucrose at a rate of $500 \mathrm{ppm} / \mathrm{fed}$ with significant differences between than all other treatments.

The different between pruning lateral branches and without pruning lateral branches were significant during the two studied seasons. No significant difference was observed in number of fruit per plant for the two seasons. The lowest early yield; total yield, average fruit weight and total yield (ton/fed.) were obtained from spraying garlic. Number of fruit per plant showed no significant differences between all treatments and pruning lateral branches during the two studied seasons.

Concerning, interaction of pruning lateral branches and natural compound and amino acids data reveal that the two treatments (spraying sucrose and spraying amino acids) gave higher early yield, total yield, average fruit weight and total yield (tonlfed.) with significant differences between them and all treatments (Table 7). The interaction of fruit yield due to spraying the suitable natural compound and amino acids might be due to the increase of the vegetative growth, dry matter and nutrient elements content of plant which consequently led to higher early and total yield. The results were agreement with those of Hahlbrock and Scheel (1989), Tawfik et 
al. (2005), Ahmad et al. (2006), Hasanein and Gaafer (2006) and Lansbergen et al. (2006)

Table 7: Effect of spraying for some natural compound, amino acids and pruning lateral branches interaction on yield and its components of cantaloupe plants grown under low tunnels conditions during $2008 / 2009$ and $2009 / 2010$ seasons.

\begin{tabular}{|c|c|c|c|c|c|c|c|c|c|c|c|}
\hline \multicolumn{2}{|c|}{ Treatments } & \multicolumn{5}{|c|}{$2008 / 2009$} & \multicolumn{5}{|c|}{$2009 / 2010$} \\
\hline Main & $\begin{array}{l}\text { sub } \\
\text { main }\end{array}$ & \begin{tabular}{|c} 
early \\
yield \\
kg \\
/plant
\end{tabular} & $\begin{array}{l}\text { No. } \\
\text { of } \\
\text { Fruit/ } \\
\text { Plant }\end{array}$ & \begin{tabular}{|c|} 
total \\
yield \\
kg/ \\
plant
\end{tabular} & $\begin{array}{c}\text { Average } \\
\text { fruit } \\
\text { weight } \\
\mathrm{g} \\
\end{array}$ & \begin{tabular}{|l} 
Total \\
yield \\
ton/ \\
fed. \\
\end{tabular} & $\begin{array}{l}\text { Early } \\
\text { yield } \\
\text { kg/ } \\
\text { plant }\end{array}$ & $\begin{array}{l}\text { No. of } \\
\text { Fruit } \\
\text { |plant }\end{array}$ & $\begin{array}{c}\text { Total } \\
\text { yield } \\
\text { kg/ } \\
\text { plant }\end{array}$ & $\begin{array}{c}\text { average } \\
\text { fruit } \\
\text { weight } \\
\text { g } \\
\end{array}$ & $\begin{array}{l}\text { total } \\
\text { yield } \\
\text { ton/ } \\
\text { fed. }\end{array}$ \\
\hline \multirow[t]{4}{*}{ PLB } & SP & 3.48 & \begin{tabular}{|l|}
10.1 \\
\end{tabular} & 7.54 & 750 & 18.85 & 3.58 & 9.9 & 7.68 & 778 & 19.2 \\
\hline & SAA & 4.05 & 9.6 & 8.23 & 854 & 20.58 & 4.19 & 9.5 & 8.31 & 874 & 20.78 \\
\hline & SS & 4.32 & 9.5 & 8.45 & 892 & 21.13 & 4.43 & 9.5 & 8.67 & 910 & 21.58 \\
\hline & C & 3.81 & 9.9 & 7.91 & 799 & 19.78 & 3.93 & 9.7 & 7.99 & 822 & 19.93 \\
\hline \multirow[t]{4}{*}{ W. PLB } & SP & 3.33 & 10.1 & 7.38 & 733 & 18.45 & 3.46 & 10.1 & 7.45 & 741 & 18.63 \\
\hline & SAA & 3.92 & 9.8 & 8.09 & 825 & 20.23 & 4.02 & 9.5 & 8.13 & 856 & 20.33 \\
\hline & sS & 4.17 & 9.6 & 8.35 & 873 & 20.88 & 4.31 & 9.5 & 8.44 & 891 & 21.10 \\
\hline & C & 3.66 & 10.2 & 7.77 & 764 & 19.43 & 3.77 & 9.9 & 7.86 & 797 & 19.65 \\
\hline LSD at & $5 \%$ & 0.06 & NS & 0.14 & 13.50 & 0.29 & 0.09 & NS & 0.12 & 17.20 & \begin{tabular}{|l|}
0.33 \\
\end{tabular} \\
\hline
\end{tabular}

PLB = pruning lateral branches W. PLB = without pruning lateral branches

$\mathrm{SP}=$ Spraying garlic extract at a rate of $200 \mathrm{ppm} / \mathrm{fed}$.

SAA = Spraying amino acid at a rate of $200 \mathrm{ppm} / \mathrm{fed}$.

$\mathrm{SS}=$ Spraying sucrose at a rate of $500 \mathrm{ppm} / \mathrm{fed}$.

$\mathrm{C}=$ without spraying (Control).

Macro and micro percentage nutrients of cantaloupe plants (leaves and stems)

Results of N, P, K, Fe and Mn contents of cantaloupe plants (leaves and stems) are presented in Table 8 .Data show that $\mathrm{N}$ and $\mathrm{P}$ contents significantly higher was without pruning lateral branches compared with pruning lateral branches in the two seasons of the study. It is clear that spraying garlic increased significantly leaves and stem contents of $N$ and $P$ over any treatment used in both seasons of study. Meanwhile using spraying sucrose increased significantly leaves and stem contents of $\mathrm{K}, \mathrm{Fe}$ and $\mathrm{Mn}$ contents compared with the other treatments and control. Similar results were obtained by Eppendorfer and Bille (1996), El-Shabasi et al. (2005), Abd ElKhair and Haggag (2007), Morsy et al. (2009) and Ayman et al. (2009).

Table (8): Effect of spraying for some natural compound, amino acids and pruning lateral branches on macro and micro nutrients of cantaloupe plants grown under low tunnels conditions during 2008/2009 and 2009/2010 seasons.

\begin{tabular}{|c|c|c|}
\hline Characters & $2008 / 2009$ & $2009 / 2010$ \\
\hline
\end{tabular}


Hasanein, N. M. et al.

\begin{tabular}{|c|c|c|c|c|c|c|c|c|c|c|c|}
\hline \multicolumn{2}{|c|}{ Treatments } & \multirow{3}{*}{$\begin{array}{c}\mathbf{N} \\
\% \\
3.0 \\
3.1\end{array}$} & \multirow{3}{*}{$\begin{array}{c}\mathbf{P} \\
\% \\
0.60 \\
0.72 \\
\end{array}$} & \multirow{3}{*}{$\begin{array}{c}\mathbf{K} \\
\% \\
5.27 \\
5.45 \\
\end{array}$} & \multirow{3}{*}{$\begin{array}{c}\begin{array}{c}\text { Fe } \\
\text { ppm }\end{array} \\
319.8 \\
335.3\end{array}$} & \multirow{3}{*}{$\begin{array}{c}\begin{array}{c}\text { Mn } \\
\text { ppm }\end{array} \\
31.3 \\
34.0\end{array}$} & \multirow{3}{*}{$\begin{array}{c}\mathbf{N} \\
\% \\
3.2 \\
3.3 \\
\end{array}$} & \multirow{3}{*}{$\begin{array}{c}\mathbf{P} \\
\% \\
0.68 \\
0.77\end{array}$} & \multirow{3}{*}{$\begin{array}{c}\mathbf{K} \\
\% \\
5.19 \\
5.36\end{array}$} & \multirow{3}{*}{$\begin{array}{c}\begin{array}{c}\text { Fe } \\
\text { ppm }\end{array} \\
310.0 \\
325.8\end{array}$} & \multirow{3}{*}{$\begin{array}{c}\begin{array}{c}\text { Mn } \\
\text { ppm }\end{array} \\
29.0 \\
31.3 \\
\end{array}$} \\
\hline & \multirow{2}{*}{\begin{tabular}{|l|l|} 
A & PLB \\
& W. PLB \\
\end{tabular}} & & & & & & & & & & \\
\hline & & & & & & & & & & & \\
\hline \multicolumn{2}{|c|}{ F. Test } & NS & * & * & * & * & NS & * & * & * & * \\
\hline \multirow[t]{4}{*}{$\mathbf{B}$} & SP & 3.3 & 0.87 & 4.86 & 283.5 & 25.0 & 3.5 & 0.94 & 4.72 & 271.0 & 22.0 \\
\hline & SAA & 3.0 & 0.57 & 5.55 & 343.0 & 35.0 & 3.2 & 0.65 & 5.51 & 335.0 & 33.0 \\
\hline & SS & 2.7 & 0.52 & 5.87 & 369.5 & 40.5 & 2.9 & 0.49 & 5.77 & 357.5 & 38.0 \\
\hline & C & 3.2 & 0.69 & 5.16 & 314.0 & 30.0 & 3.3 & 0.81 & 5.10 & 308.0 & 27.5 \\
\hline \multicolumn{2}{|c|}{ LSD at $5 \%$} & 0.1 & 0.03 & 0.25 & 7.3 & 3.6 & 0.2 & 0.06 & 0.37 & 8.4 & 2.5 \\
\hline
\end{tabular}

PLB = pruning lateral branches $\quad$ W. PLB = without pruning lateral branches

$\mathrm{SP}=$ Spraying garlic extract at a rate of $200 \mathrm{ppm} / \mathrm{fed}$.

SAA = Spraying amino acid at a rate of $200 \mathrm{ppm} / \mathrm{fed}$.

$\mathrm{SS}=$ Spraying sucrose at a rate of $500 \mathrm{ppm} / \mathrm{fed}$.

$\mathrm{C}=$ without spraying (Control).

Results of the effect of interaction between natural compound, amino acids and pruning lateral branches on some macro and micro nutrients percentage of cantaloupe plants are presented in Table 8. Both pruning lateral branches and without pruning lateral branches show higher $\mathrm{N}$ and $\mathrm{P}$ element contents by spraying garlic in the two seasons.

Table 9: Effect of spraying for some natural compound, amino acids and pruning lateral branches interaction on macro and micro nutrients of cantaloupe plants grown under low tunnels conditions during 2008/2009 and 2009/2010 seasons.

\begin{tabular}{|l|l|c|c|c|c|c|c|c|c|c|c|}
\hline \multicolumn{1}{|c|}{ Treatments } & \multicolumn{9}{|c|}{$\mathbf{2 0 0 8 / 2 0 0 9}$} & \multicolumn{6}{|c|}{$\mathbf{2 0 0 9 / 2 0 1 0}$} \\
\hline \multirow{2}{*}{ Main } & $\begin{array}{c}\text { sub } \\
\text { main }\end{array}$ & $\begin{array}{c}\mathbf{N} \\
\%\end{array}$ & $\begin{array}{c}\text { P } \\
\%\end{array}$ & $\begin{array}{c}\text { K } \\
\%\end{array}$ & $\begin{array}{c}\text { Fe } \\
\text { Ppm }\end{array}$ & $\begin{array}{c}\text { Mn } \\
\text { Ppm }\end{array}$ & $\begin{array}{c}\mathbf{N} \\
\%\end{array}$ & $\begin{array}{c}\text { P } \\
\%\end{array}$ & $\begin{array}{c}\text { K } \\
\%\end{array}$ & $\begin{array}{c}\text { Fe } \\
\text { Ppm }\end{array}$ & $\begin{array}{c}\text { Mn } \\
\text { ppm }\end{array}$ \\
\hline PLB & SP & 3.31 & 0.83 & 4.78 & 275 & 24 & 3.42 & 0.92 & 4.66 & 258 & 21 \\
& SAA & 2.93 & 0.52 & 5.43 & 337 & 33 & 3.13 & 0.61 & 5.39 & 329 & 32 \\
& SS & 2.56 & 0.39 & 5.81 & 361 & 39 & 2.84 & 0.43 & 5.73 & 353 & 37 \\
& C & 3.12 & 0.66 & 5.05 & 306 & 29 & 3.30 & 0.74 & 4.98 & 300 & 26 \\
\hline \multirow{7}{*}{ W. PLB } & SP & 3.37 & 0.91 & 4.94 & 292 & 26 & 3.49 & 0.96 & 4.77 & 284 & 23 \\
& SAA & 3.04 & 0.61 & 5.67 & 349 & 37 & 3.22 & 0.69 & 5.62 & 341 & 34 \\
& SS & 2.78 & 0.64 & 5.92 & 378 & 42 & 3.01 & 0.55 & 5.81 & 362 & 39 \\
& C & 3.25 & 0.72 & 5.26 & 322 & 31 & 3.37 & 0.87 & 5.22 & 316 & 29 \\
\hline
\end{tabular}

pruning lateral branches

$\mathrm{SP}=$ Spraying garlic extract at a rate of $200 \mathrm{ppm} / \mathrm{fed}$

SAA = Spraying amino acid at a rate of $200 \mathrm{ppm} / \mathrm{fed}$.

$\mathrm{SS}=$ Spraying sucrose at a rate of $500 \mathrm{ppm} / \mathrm{fed}$.

$\mathrm{C}=$ without spraying (Control).

Meanwhile show higher $\mathrm{K}, \mathrm{Fe}$ and $\mathrm{Mn}$ contents by using spraying sucrose compared with the other treatments during two studied seasons. The lowest nutrient elements ( $N$ and $P$ ) were found in the spraying sucrose while the lowest nutrient elements ( $\mathrm{K}, \mathrm{Fe}$ and $\mathrm{Mn}$ ) were obtained from spraying garlic. These results were confirmed two seasons and agreed with those obtained by Qualls and Haines (1991), Yu et al. (2002) and Hasanein and Gaafer (2006). 


\section{REFERENCES}

A.O.A.C. (1991). Official methods of analysis $12^{\text {th }}$ ed. Association of official analytical chemists Washington DC.

Abd-El-Khair, H. and W. M. Haggag (2007). Application of Some Egyptian Medicinal Plant Extracts Against Potato Late and Early Blights Research Journal of Agriculture and Biological Sciences, 3 (3): 166 175

Ahmad, M. E.; O. A. H. El-Zeiny and M. H. Zaky (2006). Studies of effective vitamin B1 and some amino acids on growth yield and chemical composition of bean. Egypt. J. of Appl. Sci., 21 (4B): 567-581.

Ayman, M. El-Ghamry; K. M. Abd El-Hady and M. Khalid (2009). Amino and Humic Acids promote growth, yield and disease resistance of Faba Bean cultivated in clayey Soil. Journal of Basic and Applied Sci., (3) 2: 731-739.

Balestra, G. M.; A. B. Heydari; D. C. Ceccarelli; E. C. Ovidi and A. Quattrucci (2009). Antibacterial effect of Allium sativum and ficuscarica extractson tomato bacterial pathogens. Crop Protection, (28): 807-811.

Chiappmann, H. D. and F. Pratt (1973). Method of analysis for soil .Plant and Water Calif. Univ. USA.

El- Shabasi, M. S. S.; S. M. A. Mohamed and S. A. Mahfouz (2005). Effect of foliar spray with some amino acids on growth, yield and chemical composition of garlic $6^{\text {th }}$ Arabian Conference for Hort. Ismalia Egypt, 16-24.

Eppendorfer, J. W. and S. W. Bille (1996). Free and total amino acid composition of edible parts of beans and potatoes as influenced by nitrogen fertilizations and phosphorus and potassium deficiency $\mathrm{J}$. of the Sci. of Food and Agric., 71 (4): 449-459.

Hahlbrock, K. and D. Scheel (1989). Physiology and molecular biology of phenyl propanoid metabolism. Annu. Rev. Plant Physiol. Plant Mol. Biol., 40: 347-369.

Harridy, I. M. A. (1986). Physiological studies on periwenka plant. Ph.D Thesis Fac. of Agric. Cairo Univ., Egypt.

Hasanein, N. M. and A. G. Gaafer (2006). The effect of organic manure, amino acids and sulfur on watermelon vegetative growth and yield grown under winter low plastic tunnels. Egypt J. of Appl. Sci., 21 (9): 1527-1534

Jackson, M. (1973). Soil chemical analysis prentice hall of India private Limited New Delhi 18 pp.

Joanna Majkowska-Gadomska (2009). Mineral content of melon fruit Majkowska-Gadomska J. Elementol. 14 (4): 717-727. (C.F. Computer search).

Koriesh, E. M. (1984). Effect of indole acetic acid and sodium acetate on growth and total alkahaid. J. of Agric. Res, Center Giza Egypt., 12 (4): 45-58. 
Lansbergen, L.; H. Cano and E. Lopez (2006). Viability of thinning fruits in crops of Almeria type cucumbers in the greenhouse. Agriculture, Revista-Agropecuaria. 75 (890): 862-863. (C.F. Computer search).

Lipson, D. A and T. Nasholm (2001). The unexpected versatility of plants: organic nitrogen use and availability in terrestrial ecosystems. Oecologia, 128: 305-316. (C.F. Computer search).

Mohamed, S. M; H. E. Wahba and E. A. Omar (1992). Effect of some growth regulator on the growth oil percentage and constituents of Alpinia nutans. Annals of Agric Sci. Moshtohor, 30(3): 1501-1513

Morsy, S. M.; A. E. Drgham and G. M. Mohamed (2009). Effect of garlic and onion extracts or their intercropping on suppressing damping-off and powdery mildew diseases and growth. Egypt. J. Phytopathology., 37 (1): $35-46$

Osman, A. G. (1997). Physiological studies on narcissus plant. Ph. D Thesis of Agric. Zagazig Univ., Moshtohor Egypt.

Phillips, J. D. I. (1971). Introduction of the biochemistry and physicalogy of plant growth hormones. Mc. Grow Hill Book Crop New York, London Toronto and Sydney 12pp. (C.F. Computer search).

Qualls, R. and B. L. Haines (1991). Geochemistry of dissolved organic nutrients in water percolating through a forest ecosystem. Soil Sci. Soc. Am. J. 55: 1112-1123.

Schnitzer, M., (2001). The chemistry of nitrogen in soils. Chemistry for the 21 st Century WILEY-VCH GmbH, Weinheim. 117-129.

Snedecor, G. W. and W. G. Cochran (1967). Statistical analysis $6^{\text {th }}$ ed the Lawa State Univ. Press. Amer. Lowe USA 593pp.

Sutha, R.; M. Ramiah and K. Rajappan (1998). Changes in protein and amino acid composition of tomato. Indian Phytopathology, 51 (2): 136-139.

Tawfik, E. S.; E. Hend ; E. Wahbba and A. Rashed (2005). Effect of some amino acids and sulphur fertilizers on growth and chemical composition of beans plant. J. Hort., 29 (2): 17 -37.

Yu, Z.; Q.Zhang and T. E. C. Kraus (2002). Contribution of amino compounds to dissolved organic nitrogen in forest soils. Biogeochemistry, 61: $173-198$.

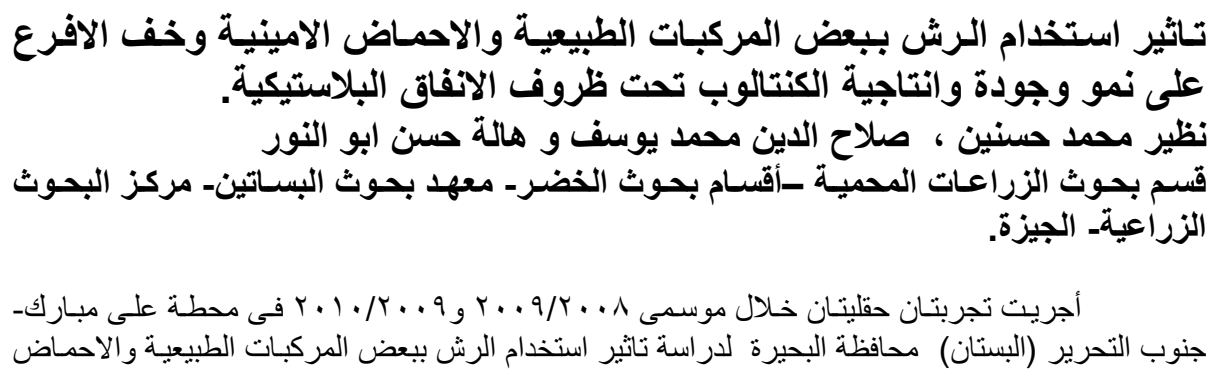




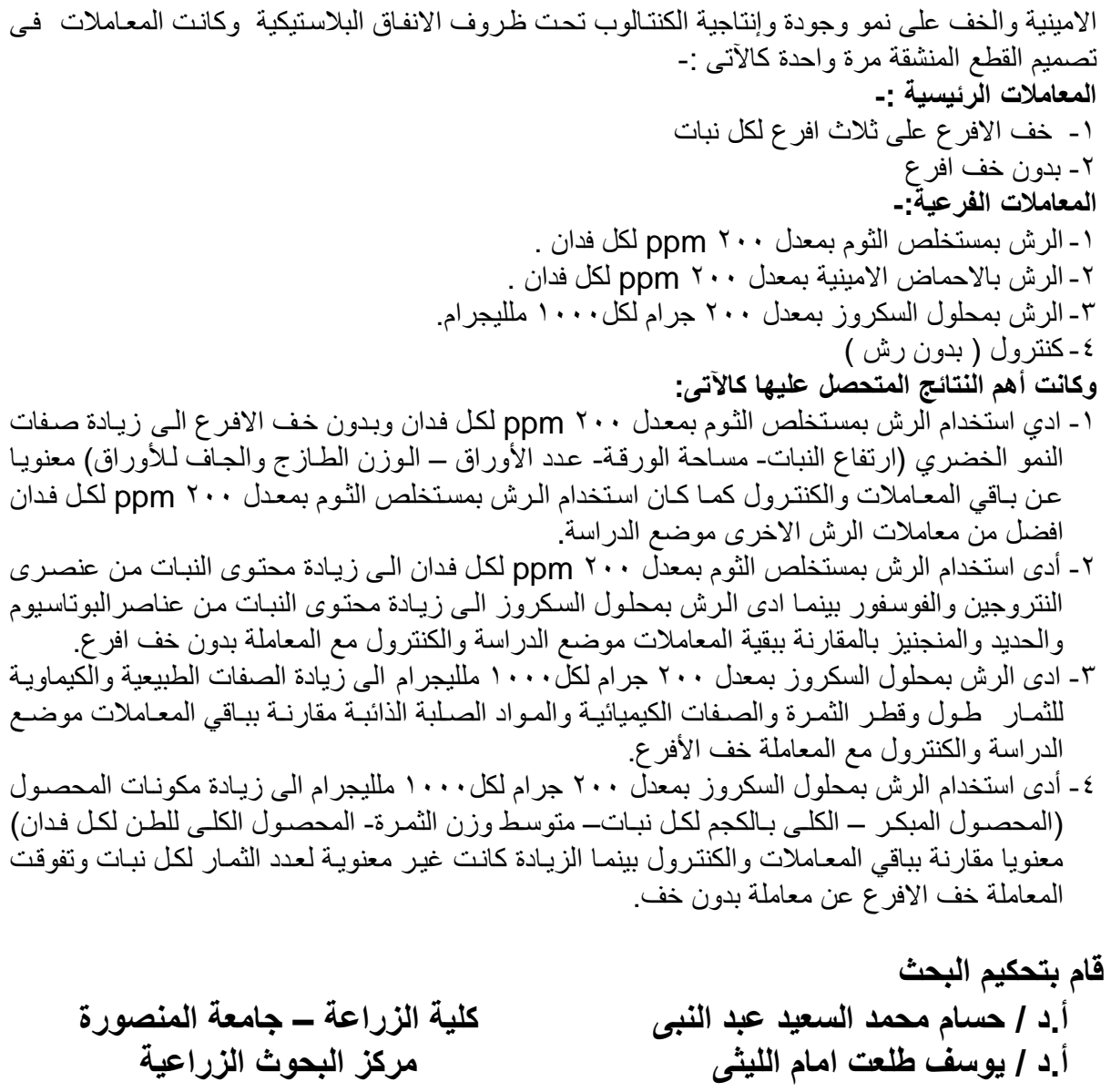

كلية الزراعة - جامعة المنصورة

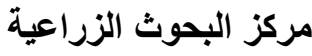

قام بتحكيم البحث

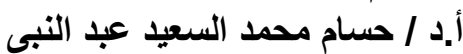

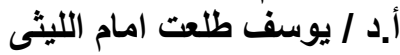

